Check for updates

Cite this: J. Mater. Chem. C, 2020, 8, 3603

DOI: $10.1039 /$ d0tc90038a

rsc.li/materials-c

\section{Correction: Room temperature discotic liquid crystalline triphenylene-pentaalkynylbenzene dyads as an emitter in blue OLEDs and their charge transfer complexes with ambipolar charge transport behaviour}

\author{
Indu Bala, ${ }^{a}$ Wan-Yun Yang, ${ }^{\text {b }}$ Santosh Prasad Gupta, ${ }^{\mathrm{c}}$ Joydip De, ${ }^{\mathrm{a}}$ \\ Rohit Ashok Kumar Yadav, ${ }^{b}$ Dharmendra Pratap Singh, ${ }^{d}$ Deepak Kumar Dubey,

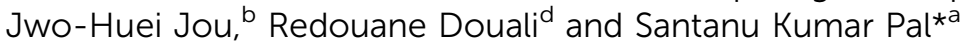

Correction for 'Room temperature discotic liquid crystalline triphenylene-pentaalkynylbenzene dyads as an emitter in blue OLEDs and their charge transfer complexes with ambipolar charge transport behaviour' by Indu Bala et al., J. Mater. Chem. C, 2019, 7, 5724-5738.

After publication, the authors found two small errors in images provided in Fig. 13e (inset) and Fig. 14e (inset) in the main paper. These errors occurred inadvertently while assembling the images for the manuscript. The corrected images are shown below (Fig. 13e (inset) is the left image; Fig. 14e (inset) is the right image):
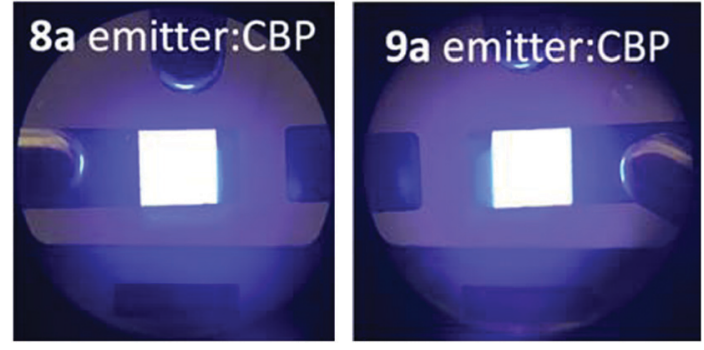

An updated graphical abstract image is also provide here:

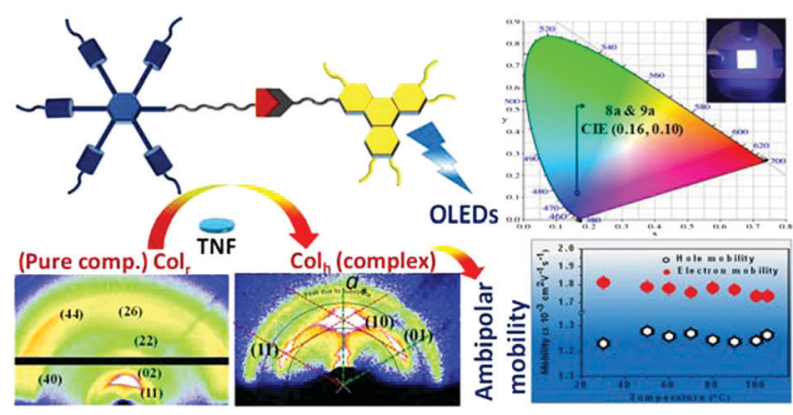

\footnotetext{
${ }^{a}$ Indian Institute of Science Education and Research (IISER) Mohali, Sector-81, SAS Nagar, Mohali 140306, India. E-mail: skpal@iisermohali.ac.in

${ }^{b}$ Department of Materials Science and Engineering, National Tsing Hua University, Hsinchu 30013, Taiwan

${ }^{c}$ Department of Physics, Patna University, Patna-800005, India

${ }^{d}$ Unité de Dynamique et Structure des Matériaux Moléculaires (UDSMM), Université du Littoral Côte d'Opale (ULCO), 50 Rue Ferdinand Buisson, 62228, Calais, France
} 
Two additional errors were also found in the Electronic Supplementary Information (ESI) Fig. S21a, f and Fig. S23d, e. The corrected ESI file has now been made available online.

The authors note that these corrections have no effect on the results reported, nor do these changes/corrections alter any of the contents and conclusions of the paper. The authors sincerely apologize for these inadvertent errors.

The Royal Society of Chemistry apologises for these errors and any consequent inconvenience to authors and readers. 